\title{
SOBREVIVÊNCIA DE BLASTOCISTOS Mus domesticus domesticus VITRIFICADOS EM MEIO CONTENDO 9,0M DE ETILENO GLICOL NA PRESENÇA DE SACAROSE
}

\author{
SURVIVAL RATE OF Mus domesticus domesticus BLASTOCYSTS VITRIFIED IN 9.0M \\ OF ETHYLENE GLYCOL WITH SUCROSE
}

\author{
Consuelo Garrastazu Paixão Côrtes ${ }^{1}$ José Luiz Rodrigues $^{2}$
}

\section{RESUMO}

Os experimentos realizados tiveram como objetivo determinar a taxa de sobrevivência in vitro $e$ in vivo de blastocistos Mus domesticus domesticus vitrificados em meio contendo 9,0M de etileno glicol e 0,3M de sacarose. As soluções testadas foram denominadas de I e IS, quando a adição do crioprotetor foi realizada em duas etapas, e II e IIS, quando a adição deste foi realizada em apenas uma etapa. Na solução de vitrificação $I$, foi realizada inicialmente uma desidratação prévia dos embriões por um período de 2 minutos em solução de 1,8M de EG em $P B S+$ $6 \%$ de BSA e, logo após, eles foram transferidos, por um período de 30 segundos, para a solução composta por uma associação de 9,0M de EG em PBS + 6\% de BSA, antes da imersão em nitrogênio líquido. Na solução de vitrificação $I S$, o procedimento foi idêntico ao da solução I, apenas com o acréscimo de 0,3M de sacarose às soluções crioprotetoras. $\mathrm{Na}$ solução de vitrificação II, os embriões foram expostos diretamente a uma solução composta por uma associação de 9,0M de EG em PBS + 6\% de BSA, onde permaneciam por 30 segundos antes da imersão em nitrogênio líquido. Na solução de vitrificação IIS, o procedimento foi idêntico ao da solução II, apenas com a adição de $0,3 \mathrm{M}$ de sacarose à solução crioprotetora. No experimento I, foi determinada a taxa de sobrevivência de 299 blastocistos após a exposição às soluções crioprotetoras, não sendo observada diferença estatística entre os tratamentos e o grupo controle. O experimento II permitiu avaliar a sobrevivência embrionária in vitro (taxa de eclosão) após a vitrificação de 330 blastocistos, onde as soluções I e IS foram estatisticamente superiores às demais, apresentando taxas de eclosão de 49 e 40\%, respectivamente. No experimento III, realizou-se a transferência de 141 blastocistos vitrificados, após a exposição às soluções I e IS, para fêmeas receptoras. Não houve diferenças estatísticas entre as taxas de sobrevivência embrionária determinadas aos 14 dias de prenhez, tanto para implantações $(37$ e $32 \%$ ) quanto para fetos $(27 e$ $27 \%)$, respectivamente. A presença de sacarose na solução de vitrificação não proporcionou uma maior sobrevivência de blastocistos Mus domesticus domesticus vitrificados em uma solução contendo 9,0M EG.
Palavras-chave: roedores, embriões, crioconservação.

\section{SUMMARY}

The aim of these experiments was to determine the in vitro and in vivo survivability of Mus domesticus domesticus blastocysts, following exposure and vitrification in modified PBS solution containing 9.0M of ethylene glycol (EG) and $0.3 \mathrm{M}$ of sucrose. The cryoprotectant solutions were denominated I (without sucrose) and IS (with sucrose) when the embryo exposure to the solutions has been done in two steps. First the embryos were exposed to a modified PBS solution with 1,8M EG $+6 \%$ BSA during 2 minutes, and after they were transferred to straws containing modified PBS plus 9.OM EG +6\% BSA and after 30 seconds immersed into liquid nitrogen. The others cryoprotectant solutions, denominated II (without sucrose) and IIS (with sucrose) the embryos were exposed directly to the $9.0 \mathrm{M}$ $E G$ and after 30 seconds plunged into the liquid nitrogen. In the first experiment a survival rate of 299 blastocysts was observed following exposure to cryoprotectant solutions. There was no statistical diference among control and treatment groups. The experiment II allowed to evaluate the in vitro embryo survival, after vitrification of 330 blastocysts, the cryoprotectant solutions $I$ and IS determinated statistically different $(p<0.05)$ embryo survival rates, showing in vitro hatching rates of 49 and $40 \%$, respectively. In experiment 3,141 blastocysts were exposed to the cryoprotectant solutions I and IS, vitrified and then transferred into the recipients. The number of implantations and fetuses were observed after 14 days of gestation. There was no statistical difference ( $p>0.05$ ) among the solutions I and IS in the number of implantations (37 and 32\%) and fetuses (27 and 27\%), respectively. The survival rate of Mus domesticus domesticus blastocysts did not improve with the addition of sucrose to the vitrification solution containing 9.0M of EG.

Key words: rodent, embryo, cryopreservation.

\footnotetext{
${ }^{1}$ Médico Veterinário, aluno do CPG em Zootecnia, Universidade Federal do Rio Grande do Sul (UFRGS), bolsista - CNPq.

${ }^{2}$ Médico Veterinário, Professor Titular, Departamento de Patologia Clínica, UFRGS, pesquisador CNPq. CP 15004, 91501-970, Porto Alegre-RS. E-mail: jlr@ orion.ufrgs.br. Autor para correspondência.
} 


\section{INTRODUÇÃO}

Os avanços tecnológicos da genética molecular, em especial na produção de animais transgênicos e mutantes na espécie Mus domesticus domesticus, permitem, segundo ROBERTSON (1991), o desenvolvimento de novos modelos animais para estudos básicos e aplicados, tanto das funções biológicas normais quanto dos processos patológicos. $\mathrm{O}$ alto custo de manutenção e da reprodução dessas linhagens em biotérios, o que poderia tornar-se economicamente inviável, criou a necessidade da organização de bancos de embriões. SCHMIDT et al. (1996) citam que mais de 300 genótipos de roedores são distribuídos mundialmente para pesquisas nas áreas biomédicas. Segundo RALL (1987), a criopreservação pelo método da vitrificação oferece vantagens, quando comparada a outros procedimentos, que permitem ou exigem a cristalização da suspensão por não requerer equipamentos, nem o controle das curvas de resfriamento e de aquecimento, normalmente necessários nos procedimentos convencionais.

Entre os diferentes crioprotetores empregados na vitrificação, o etileno glicol (EG) apresenta baixa toxicidade e baixo peso molecular, o que facilita sua rápida permeação para o interior das células durante um curto período de exposição (KASAI et al., 1990).

Rotinas experimentais de criopreservação de embriões vêm sendo realizadas pelo Laboratório de Embriologia e Biotécnicas de Reprodução da Faculdade de Veterinária da UFRGS, desde o ano de 1984. Em experimentos desenvolvidos em vitrificação utilizando o glicerol e o EG como crioprotetores, CHRISTMANN (1989), LOPES (1989), HÖTZEL (1991), PALHA (1992), BERTOLINI (1994) e AGUIAR (1996) observaram o desenvolvimento de fetos após a transferência de embriões no estádio de mórula $(34 \%, 47 \%, 34 \%, 42 \%, 27 \%$ e $46 \%$, respectivamente) que não diferiram, estatisticamente, dos seus respectivos grupos controle. Ao contrário, quando vitrificaram blastocistos, os mesmos índices de desenvolvimento in vivo não puderam ser obtidos por CHRISTMANN (1989), LOPES (1989), BERTOLINI (1994) e AGUIAR (1996), que registraram taxas de $22 \%, 19 \%$, 9\% e $16 \%$, respectivamente. Apesar de HÖTZEL (1991) e PALHA (1992) terem obtido taxas de $39 \%$ e $41 \%$ de desenvolvimento embrionário até fetos, as quais não diferiram dos seus respectivos grupos controles, esses resultados de sobrevivência ainda não podem ser considerados satisfatórios para a rotina da crioconservação de mórulas e blastocistos Mus domesticus domesticus.
Os experimentos de vitrificação ainda não permitiram a descrição de uma adequada composição da solução crioprotetora, que propicie taxas de sobrevivência embrionária após a transferência para as receptoras em percentuais acima dos 70\%. A eleição do estádio de blastocisto para utilização nesses experimentos foi determinada pela necessidade de procurar-se alcançar percentuais de sobrevivência in vivo similares ou superiores aos já obtidos com o estádio de mórula compacta.

Os experimentos realizados tiveram por objetivo determinar o efeito da adição de $0,3 \mathrm{M}$ de sacarose às soluções de vitrificação, sobre a viabilidade in vitro e in vivo de blastocistos Mus domesticus domesticus.

\section{MATERIAL E MÉTODOS}

Doadoras e receptoras Mus domesticus domesticus da linhagem suíça albina CF1, com idade variando entre 6 e 8 semanas, foram submetidas ao tratamento superovulatório, recebendo uma aplicação intraperitonial de 10 UI de eCG e, 46 horas após, 10 UI de hCG, pela mesma via. Blastocistos foram colhidos na manhã do $4^{\circ}$ dia, após a lavagem do lúmen dos cornos uterinos utilizando uma solução de PBS modificada (DULBECCO \& VOGT, 1954) + $20 \%$ de soro bovino inativado (SBI). Os embriões classificados morfologicamente como excelentes (grau 1) foram submetidos à vitrificação.

Para a vitrificação dos embriões, foi utilizada uma solução de PBS acrescida de $6 \%$ de BSA, com diferentes concentrações de EG, de acordo com o tratamento proposto. As soluções testadas foram denominadas de I e IS, quando a adição do crioprotetor foi realizada em duas etapas; II e IIS, quando a adição deste foi realizada em apenas uma etapa. $\mathrm{Na}$ solução de vitrificação I, foi realizada, inicialmente, uma desidratação prévia dos embriões por um período de 2 minutos em solução de 1,8M de EG em PBS $+6 \%$ de BSA e, logo após, eles foram transferidos para a solução composta por uma associação de 9,0M de $\mathrm{EG}$ em $\mathrm{PBS}+6 \%$ de BSA, por um período de 30 segundos antes da imersão em nitrogênio líquido. Na solução de vitrificação IS, o procedimento foi idêntico ao da solução I, apenas com o acréscimo de $0,3 \mathrm{M}$ de sacarose em cada etapa da solução.

Na solução de vitrificação II, os embriões foram expostos diretamente a uma solução composta por uma associação de 9,0M de EG em PBS + 6\% de BSA, onde permaneciam por 30 segundos antes da imersão das palhetas em nitrogênio líquido. $\mathrm{Na}$ solução de vitrificação IIS, o procedimento foi idêntico ao da solução II, apenas com a adição de $0,3 \mathrm{M}$ de sacarose. 
No teste de toxicidade (experimento I), os blastocistos foram expostos às soluções de vitrificação, conforme os tratamentos propostos e, em seguida, submetidos às soluções de retirada do crioprotetor. Posteriormente, foram cultivados em meio de Whitten em estufa de $\mathrm{CO}_{2}$ a $37,5^{\circ} \mathrm{C}$ e $100 \%$ de umidade relativa do ar. A viabilidade embrionária foi determinada pela taxa de eclosão após 48 horas de cultivo in vitro.

No experimento II, os blastocistos, em número de 10 a 15, após serem expostos às soluções de pré-equilíbrio, foram pipetados para o interior de uma palheta francesa de $0,25 \mathrm{~m} \ell$, contendo aproximadamente $0,1 \mathrm{~m} \ell$ da solução de vitrificação, sendo as palhetas, após 30 segundos, imersas em nitrogênio líquido.

Para a desvitrificação dos embriões, as palhetas foram expostas ao ar por 10 segundos e, em seguida, imersas em banho-maria a $20^{\circ} \mathrm{C}$ por 20 segundos. Foram testados dois procedimentos de retirada do crioprotetor, sendo: a) PBS: PBS + 0,4\% de BSA; e b) SAC: $1,8 \mathrm{M}$ de EG + 0,4\% de BSA + $1,0 \mathrm{M}$ de sacarose (2 minutos). Após a desvitrificação, os blastocistos foram cultivados em meio de Whitten em estufa de $\mathrm{CO}_{2}$ a $37,5^{\circ} \mathrm{C}$ e $100 \%$ de umidade relativa do ar. A viabilidade embrionária foi determinada pela taxa de eclosão após 48 horas de cultivo in vitro. No experimento III, foi realizada a avaliação in vivo dos blastocistos desvitrificados; estes foram classificados morfologicamente após o cultivo in vitro de uma hora em estufa de $\mathrm{CO}_{2}$ a $37,5^{\circ} \mathrm{C}$ com $100 \%$ de umidade relativa do ar. De 6 a 8 embriões classificados morfologicamente como excelentes e bons foram transferidos para o corno uterino direito de fêmeas receptoras, no terceiro dia de pseudoprenhez. Transcorridos 14 dias da transferência, as fêmeas foram sacrificadas e quantificado o número de implantações e fetos. Como controle, foram transferidos para o corno esquerdo das receptoras blastocistos frescos colhidos na manhã do quarto dia de prenhez.

Para a análise dos dados obtidos, foi empregado o teste QuiQuadrado e a existência de significância, nesse teste, determinou a necessidade da análise de resíduos ajustados para comprovar quais tratamentos foram estatisticamente diferentes entre si $(\alpha=0,05)$.

\section{RESULTADOS E DISCUSSÃO}

A tabela 1 mostra que não houve diferença significativa entre os tratamentos no experimento I. Os resultados observados indicam que os blastocistos, quando expostos aos tratamentos propostos, não sofreram danos em decorrência da toxicidade. TACHIKAWA et al. (1993) citam que a simples exposição dos embriões às soluções de vitrificação reduz a sobrevivência embrionária e que a toxicidade das soluções depende da permeação do crioprotetor.

Não foi observada a ocorrência de choque osmótico nas células embrionárias após a direta exposição dos blastocistos à solução de 9,0M de EG, confirmando as afirmações de KASAI et al. (1990) sobre a permeabilidade desse crioprotetor às membranas celulares. Apesar dessa característica do EG, MAHMOUDZADEH $\boldsymbol{e t}$ al. (1995) citam a necessidade de uma desidratação dos embriões bovinos, produzidos in vitro, prévia à exposição à solução composta por etileno glicol, ficoll e sacarose (EFS), para que a vitrificação proporcione viabilidade em-
Tabela 1 - Viabilidade in vitro de blastocistos após o equilíbrio nas soluções criopatogênicas.

\begin{tabular}{|c|c|c|c|c|c|}
\hline \multirow[t]{2}{*}{ Tratamento } & \multirow[t]{2}{*}{$\begin{array}{l}\text { Soluções } \\
\text { crioprotetoras }\end{array}$} & \multirow[t]{2}{*}{$\begin{array}{l}\text { Retirada do } \\
\text { Crioprotetor }\end{array}$} & \multirow{2}{*}{$\begin{array}{c}\text { Embriões } \\
\text { Expostos } \\
\mathrm{N}\end{array}$} & \multicolumn{2}{|c|}{ Eclosão } \\
\hline & & & & $\mathrm{N}$ & $\%$ \\
\hline I & $\begin{array}{l}1,8 \mathrm{M} \text { de } \mathrm{EG} \text { em PBS }+ \\
6 \% \text { de BSA ( } 2 \text { minutos) }\end{array}$ & a)PBS + 0,4\% de BSA; & 33 & 30 & 91 \\
\hline \multirow{3}{*}{ IS } & $\begin{array}{l}+ \\
9,0 \mathrm{M} \text { de } \mathrm{EG} \text { em PBS + } \\
6 \% \text { de BSA }\end{array}$ & $\begin{array}{l}\text { b) } 1,8 \mathrm{M} \text { de } \mathrm{EG}+0,4 \% \\
\text { de } \mathrm{BSA}+1,0 \mathrm{M} \text { de } \\
\text { sacarose ( } 2 \text { minutos })\end{array}$ & 31 & 27 & 87 \\
\hline & $\begin{array}{l}1,8 \mathrm{M} \text { de } \mathrm{EG} \text { em } \mathrm{PBS}+ \\
6 \% \text { de BSA ( } 2 \text { minutos) }\end{array}$ & a)PBS $+0,4 \%$ de BSA; & 42 & 40 & 95 \\
\hline & $\begin{array}{l}+9,0 \mathrm{M} \text { de } \mathrm{EG} \text { em PBS } \\
+6 \% \text { de } \mathrm{BSA}+0,3 \mathrm{M} \\
\text { sacarose }\end{array}$ & $\begin{array}{l}\text { b) } 1,8 \mathrm{M} \text { de } \mathrm{EG}+0,4 \% \\
\text { de } \mathrm{BSA}+1,0 \mathrm{M} \text { de } \\
\text { sacarose ( } 2 \text { minutos })\end{array}$ & 39 & 37 & 95 \\
\hline \multirow[t]{2}{*}{ II } & $\begin{array}{l}9,0 \mathrm{M} \text { de EG em PBS } \\
+6 \% \text { de BSA }\end{array}$ & a)PBS + 0,4\% de BSA; & 41 & 38 & 92 \\
\hline & & $\begin{array}{l}\text { b) } 1,8 \mathrm{M} \text { de } \mathrm{EG}+0,4 \% \\
\text { de BSA }+1,0 \mathrm{M} \text { de } \\
\text { Sacarose ( } 2 \text { minutos })\end{array}$ & 36 & 34 & 94 \\
\hline \multirow[t]{2}{*}{ IIS } & $\begin{array}{l}9,0 \mathrm{M} \text { de } \mathrm{EG} \text { em PBS + } \\
6 \% \text { de } \mathrm{BSA}+0,3 \mathrm{M}\end{array}$ & a)PBS + 0,4\% de BSA; & 41 & 37 & 90 \\
\hline & sacarose & $\begin{array}{l}\text { b) } 1,8 \mathrm{M} \text { de } \mathrm{EG}+0,4 \% \\
\text { de BSA + } 1,0 \mathrm{M} \text { de } \\
\text { sacarose ( } 2 \text { minutos). }\end{array}$ & 36 & 33 & 92 \\
\hline
\end{tabular}


brionária. Os autores observaram que a taxa de sobrevivência dos blastocistos, após a vitrificação, foi aumentada significativamente de 58\% (43/74) para $75 \%$ (59/79) pela adição da solução de vitrificação em duas etapas. De modo similar, ZHU et al. (1993) obtiveram taxas de sobrevivência de 94\% (64/68) e de $87 \%$ (58/67) para blastocistos expandidos de camundongo vitrificados após a exposição a $10 \mathrm{ou}$ $20 \%$ de EG, respectivamente, por 5 minutos e, posteriormente, expostos à solução EFS por 30 segundos. A sobrevivência foi determinada pela taxa de blastocistos reexpandidos em 48 horas de cultivo. Os resultados de toxicidade das soluções crioprotetoras obtidos no experimento I permitiram a utilização dessas soluções nos demais experimentos.

$\mathrm{O}$ experimento II avaliou a viabilidade embrionária in vitro através da taxa de eclosão após a desvitrificação e cultivo por 48 horas. Após a desvitrificação, foi realizada a retirada do crioprotetor diretamente em uma solução de PBS + 0,4\% BSA ou em 1,8M de EG + 0,4 BSA + 1,0M de sacarose.

A tabela 2 mostra que os tratamentos I-a) e IS-b) foram superiores, estatisticamente, em relação aos demais, apresentando taxas de eclosões de 49 e $40 \%$ respectivamente.

Tabela 2 - Sobrevivência de blastocistos de Mus domesticus domesticus após a desvitrificação e cultivo in vitro por 48 horas.

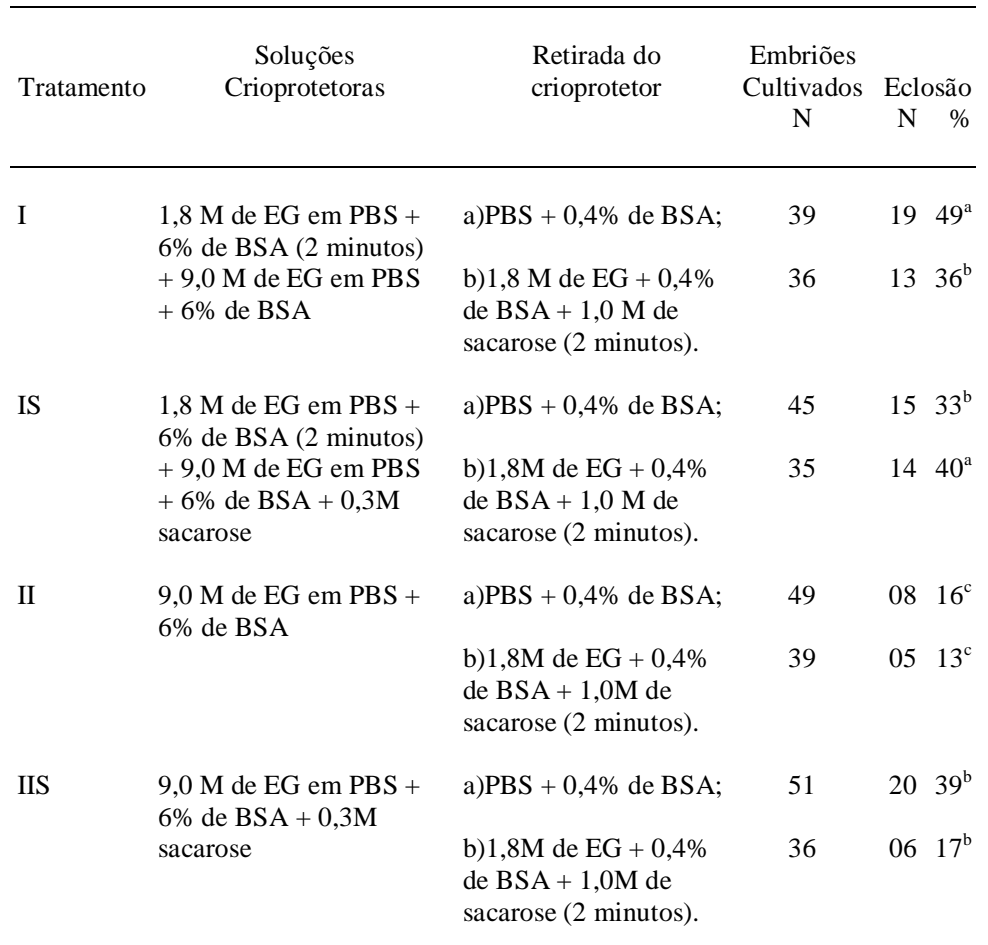

a,b,c números seguidos de letras desiguais na coluna diferem estatisticamente pelo teste $\chi^{2}(\mathrm{p}<0.05)$
Considerando as soluções de vitrificação que contém sacarose, IS e IIS, observou-se não haver diferença significativa entre esses tratamentos quando a retirada do crioprotetor foi realizada diretamente em solução de PBS. Ao passo que a presença de sacarose na solução permitiu que a taxa de eclosão, observada no tratamento IS, fosse estatisticamente superior ao tratamento IIS. Comparando-se as taxas de sobrevivência embrionária obtidas nos tratamentos que utilizaram duas etapas de adição do crioprotetor (I e IS), verificou-se que, uma vez incluída a sacarose na solução de vitrificação, faz-se necessária a presença desse polímero de alto peso molecular na solução de retirada do crioprotetor. Os resultados de sobrevivência embrionária, observados na tabela 2 , mostram que quando for realizada a adição em etapas do crioprotetor sem sacarose (I), a retirada deste deve ser feita diretamente em solução de PBS.

ALI \& SHELTON (1993b) observaram, após a vitrificação de blastocistos de camundongo em VS11 (6,0M de EG + 1,8M de glicerol), que não houve diferença estatística entre os métodos de retirada do crioprotetor, ou seja, diretamente em solução isotônica ou em $1 \mathrm{M}$ de sacarose, obtendo, respectivamente, 93 e $79 \%$ de taxas de eclosão após o cultivo in vitro. Similarmente, MAHMOUDZADEH $\boldsymbol{e t}$ al. (1995) concluíram que não há necessidade da presença de sacarose na solução de retirada do crioprotetor após a vitrificação em EFS de embriões bovinos produzidos in vitro. Os autores obtiveram taxas de eclosão para blastocistos de $40 \%$ (30/75), 44\% (32/72) e 30\% (21/71), respectivamente, após a exposição dos embriões, para a retirada do crioprotetor, a concentrações de sacarose de $0 \%, 0,25 \%$ e $0,5 \%$. Segundo KASAI $\boldsymbol{e t}$ al. (1990), a sacarose associada a uma macromolécula pode reduzir a toxicidade do etileno glicol pela rápida redução da quantidade de água intracelular. Isso também foi reportado por SZÉLL \& SHELTON (1986) quando utilizaram glicerol como crioprotetor. Pode-se observar, neste experimento, que a presença da sacarose na solução de vitrificação $(9,0 \mathrm{M})$ não foi imprescindível para o incremento da sobrevivência embrionária in vitro, quando utilizada a adição do crioprotetor em etapas. A tabela 2 mostra que não há 
diferença significativa entre os tratamentos I e IS, desde que se utilize o método adequado de retirada do crioprotetor, ou seja, no tratamento I, diretamente em uma solução de PBS e no IS, na presença de sacarose. Nos tratamentos, nos quais os embriões foram expostos diretamente a uma solução de $9,0 \mathrm{M}$ (II e IIS), a inclusão da sacarose na solução de vitrificação proporcionou uma taxa de eclosão in vitro superior à observada com os embriões que foram expostos à solução na ausência da referida substância. Esses achados corroboram os resultados obtidos por KASAI et al. (1990) que, empregando a vitrificação, expuseram mórulas de camundongo em solução de EF e EFS por 5 minutos antes da imersão em nitrogênio líquido, obtendo $52 \%$ (26/50) e $98 \%$ (49/50), respectivamente, de desenvolvimento in vitro até o estádio de blastocistos expandido. A sacarose adicionada à solução de vitrificação no tratamento IIS, provavelmente, promoveu uma desidratação mais adequada, impedindo uma maior permeação do EG, evitando, assim, os efeitos tóxicos da solução. Foi possível observar que a concentração de 9,0M de EG, ou seja, 50\% de EG, não se apresentou tóxica aos blastocistos (Tabela 1) e que também conferiu uma proteção adequada às células embrionárias após serem submetidas ao processo de vitrificação. Comparando o tratamento I com o II (Tabela 2), pode-se observar que a adição em etapas do crioprotetor proporcionou taxas de sobrevivência embrionária estatisticamente superiores à adição em uma única etapa (tratamento II), sendo esses resultados independentes do método utilizado para a retirada do crioprotetor. Dados similares foram obtidos por ZHU et al. (1993), quando vitrificaram blastocistos expandidos de camundongo em EFS, empregando uma etapa com $10 \%$ de EG antes da exposição à solução de vitrificação. Os autores citam que a permeação de EG, possivelmente para o interior da blastocele, durante um pré-tratamento com uma solução diluída de EG evitou a formação letal de cristais de gelo, enquanto que um menor tempo de exposição dos embriões à solução EFS reduziu a injúria tóxica. À mesma conclusão chegaram MAHMOUDZADEH et al. (1995), que, após vitrificarem blastocistos bovinos produzidos in vitro, observaram 38\% (30/79) de eclosão em 72 horas de cultivo no grupo de embriões onde a adição do crioprotetor foi realizada em etapas, e somente $19 \%$ (14/74) no grupo onde os embriões foram expostos diretamente em EFS.

A vitrificação de blastocistos Mus

domesticus domesticus com as soluções propostas neste experimento, provavelmente, determinou danos irreversíveis na organização do citoesqueleto das células embrionárias. De acordo com o já referido por OVERSTRÖM et al. (1993), devido à exposição desses embriões a soluções muito concentradas de crioprotetor. Essa hipótese pode ser sustentada pelo fato de que no experimento I, como mostra a tabela 1 , as taxas de eclosão observadas não diferiram estatisticamente dos seus respectivos grupos controles. A utilização de estabilizadores de citoesqueleto como aqueles preconizados por DOBRINSKY (1996) poderiam ser testados como alternativa para incrementar as taxas de eclosão dos embriões vitrificados nestas soluções, pois estas não apresentaramse tóxicas às células embrionárias no experimento I. No experimento III, foi realizada a avaliação do desenvolvimento in vivo, após a transferência dos blastocistos vitrificados com os protocolos dos tratamentos testados, visto terem sido observadas, nesses grupos de embriões, taxas de eclosões superiores, estatisticamente, aos demais tratamentos do experimento II (in vitro). Após a desvitrificação, os embriões foram cultivados por uma hora em estufa de incubação a uma temperatura de $37,5^{\circ} \mathrm{C}$ em meio de PBS e, posteriormente à análise da morfologia, os considerados excelentes foram transferidos para receptoras no terceiro dia da pseudoprenhez. Transferiu-se um total de 141 blastocistos para 40 receptoras nos tratamentos I-a) e IS-b), não havendo diferença estatística entre as taxas de prenhez obtidas, de $59 \%(10 / 17)$ e de $43 \%$ (10/23), respectivamente. Os resultados de implantação após a transferência dos blastocistos estão apresentados na tabela 3. As taxas de sobrevivência embrionária, observadas com os blastocistos submetidos à vitrificação com os dois tratamentos testados, foram semelhantes e também não diferiram estatisticamente do seu grupo controle $(\mathrm{p}<0,05)$.

Esses resultados foram similares aos de ALI \& SHELTON (1993a), que obtiveram uma taxa de implantação de 25\% (49/193) para blastocistos utilizando a solução VS11, composta de 6,0M de EG $+1,8 \mathrm{M}$ de glicerol, sendo realizada a retirada do crioprotetor em 1,0M de sacarose. Esses autores verificaram que, nos estudos in vitro, a remoção do crioprotetor utilizando solução contendo sacarose não seria necessária para a sobrevivência de embriões de 8 células e blastocistos de camundongos; enquanto que para proporcionar o desenvolvimento in vivo desses embriões, a presença de sacarose na remoção do crioprotetor foi necessária, alcançando um número estatisticamente superior de fetos. Os autores observaram ainda que os embriões vitrificados produzem um maior número de implantações e o mesmo número de fetos vivos que o observado no grupo controle não tratado, quando a solução de vitrificação é removida com sacarose. 
Tabela 3 - Implantações após a transferência dos blastocistos.

\begin{tabular}{|c|c|c|c|c|c|c|c|c|}
\hline \multirow[t]{3}{*}{ Tratamento } & \multicolumn{2}{|c|}{ Soluções crioprotetoras } & \multicolumn{3}{|c|}{ Desvitrificados } & \multicolumn{3}{|c|}{ Controle } \\
\hline & \multirow{2}{*}{ Crioprotetor } & \multirow{2}{*}{$\begin{array}{l}\text { Retirada do } \\
\text { crioprotetor }\end{array}$} & \multirow{2}{*}{$\begin{array}{c}\text { Transf. } \\
\mathrm{N}\end{array}$} & \multicolumn{2}{|c|}{ Implantações } & \multirow{2}{*}{$\frac{\text { Transf. }}{\mathrm{N}}$} & \multicolumn{2}{|c|}{ Implantações } \\
\hline & & & & $\mathrm{N}$ & $\%$ & & $\mathrm{~N}$ & $\%$ \\
\hline I & $\begin{array}{l}1,8 \mathrm{M} \text { de } \mathrm{EG} \text { em PBS }+ \\
6 \% \text { de BSA }(2 \text { minutos })+ \\
9,0 \mathrm{M} \text { de EG em PBS }+ \\
6 \% \text { de BSA }\end{array}$ & $\begin{array}{l}\text { PBS + 0,4\% } \\
\text { de BSA; }\end{array}$ & 70 & 26 & 37 & 76 & 31 & 41 \\
\hline IS & $\begin{array}{l}1,8 \mathrm{M} \text { de EG em PBS + } \\
6 \% \text { de BSA ( } 2 \text { minutos })+ \\
9,0 \mathrm{M} \text { de } \mathrm{EG} \text { em PBS }+ \\
6 \% \text { de BSA }+0,3 \mathrm{M} \\
\text { sacarose }\end{array}$ & $\begin{array}{l}1,8 \mathrm{M} \text { de } \mathrm{EG} \\
+0,4 \% \text { de } \\
\text { BSA }+1,0 \mathrm{M} \text { de } \\
\text { sacarose }(2 \\
\text { minutos }) .\end{array}$ & 71 & 23 & 32 & 75 & 27 & 36 \\
\hline
\end{tabular}

trole. Com base nessa informação, os resultados sugerem que os tratamentos utilizados neste experimento conferiram uma proteção similar às células do embrioblasto e do trofoectoderma. Esses dados divergem do que foi reportado por AGUIAR (1996) que, utilizando uma VSIII composta por $9,0 \mathrm{M}$ de EG, obteve $50 \%$ (30/60) de implantações após a transferência dos blastocistos e $30 \%(18 / 60)$ de fetos. O diferencial de $20 \%$ de mortalidade

DINNYÉS et al. (1995) citam que altas taxas de desenvolvimento in vitro não asseguraram as mesmas taxas de desenvolvimento in vivo, após a transferência embrionária, devido à extensão da mortalidade pós-implantação e da taxa de desenvolvimento de fetos que variaram conforme o genótipo do embrião. Esses autores contrariam as afirmações de WHITTINGHAM (1978), que considera haver uma boa correlação entre a sobrevivência in vitro e a viabilidade embrionária in vivo seguida da transferência após a descongelação.

A tabela 4 mostra que não foi verificada diferença estatística significativa entre os tratamentos e também dos tratamentos com os grupos con-

Tabela 4 - Desenvolvimento dos blastocistos desvitrificados em fetos.

\begin{tabular}{|c|c|c|c|c|c|c|c|c|}
\hline \multirow{3}{*}{ Tratamento } & \multicolumn{2}{|c|}{ Soluções crioprotetoras } & \multicolumn{3}{|c|}{ Desvitrificados } & \multicolumn{3}{|c|}{ Controle } \\
\hline & \multirow{2}{*}{ Crioprotetor } & \multirow{2}{*}{$\begin{array}{l}\text { Retirada do } \\
\text { Crioprotetor }\end{array}$} & \multirow{2}{*}{$\frac{\text { Transf. }}{\mathrm{N}}$} & \multicolumn{2}{|c|}{ Fetos } & \multirow{2}{*}{$\frac{\text { Transf. }}{\mathrm{N}}$} & \multicolumn{2}{|c|}{ Fetos } \\
\hline & & & & $\mathrm{N}$ & $\%$ & & $\mathrm{~N}$ & $\%$ \\
\hline I & $\begin{array}{l}1,8 \mathrm{M} \text { de EG em PBS }+ \\
6 \% \text { de BSA }(2 \text { minutos })+ \\
9,0 \mathrm{M} \text { de EG em PBS }+ \\
6 \% \text { de BSA }\end{array}$ & $\begin{array}{l}\text { PBS }+0,4 \% \\
\text { de BSA; }\end{array}$ & 70 & 19 & 27 & 76 & 26 & 34 \\
\hline IS & $\begin{array}{l}1,8 \mathrm{M} \text { de EG em PBS }+ \\
6 \% \text { de BSA }(2 \text { minutos })+ \\
9,0 \mathrm{M} \text { de } \mathrm{EG} \text { em PBS }+ \\
6 \% \text { de } \mathrm{BSA}+0,3 \mathrm{M} \\
\text { sacarose }\end{array}$ & $\begin{array}{l}1,8 \mathrm{M} \text { de EG } \\
+0,4 \% \text { de } \\
\text { BSA }+1,0 \mathrm{M} \text { de } \\
\text { sacarose }(2 \\
\text { minutos }) .\end{array}$ & 71 & 19 & 27 & 75 & 23 & 31 \\
\hline
\end{tabular}

embrionária precoce poderia ser atribuído à ocorrência de um maior número de lesões nas células do embrioblasto em relação às do trofoectoderma. Essas observações também foram reportadas por RALL $\boldsymbol{e t}$ al. (1987), RALL (1987), KASAI et al. (1992a,b).

A homogeneidade obtida entre os resultados dos tratamentos testados e o grupo controle, tanto em relação às taxas de prenhez e de implantações, como às taxas de desenvolvimento in vivo, indica que houve uma adequada avaliação morfológica dos embriões, prévia à transferência para as receptoras. Ao mesmo tempo, esses resultados vem corroborar a correlação existente entre a sobrevivência embrionária in vitro e in vivo reportada por WHITTINGHAN (1978).

\section{CONCLUSÕES}

A desidratação prévia dos embriões promove um aumento da taxa de eclosão de blastocistos Mus domesticus domesticus vitrificados em 9,0M de EG. A adição de $0,3 \mathrm{M}$ de sacarose às soluções de vitrificação e de retirada do crioprotetor não altera as taxas de sobrevivência in vitro e in vivo dos blastocistos.

\section{FONTES DE AQUISIÇÃO}

\footnotetext{
${ }^{a}$ Etileno glicol p.a.- Reagen.

${ }^{\mathrm{b}}$ Sacarose p.a - Merk.
}

Ciência Rural, v. 30, n. 3, 2000. 


\section{REFERÊNCIAS BIBLIOGRÁFICAS}

AGUIAR, P.R.L. Sobrevivência de embriões Mus domesticus domesticus vitrificados em diferentes soluções de criopreservação. Porto Alegre, 1996. 148p. Dissertação (Mestrado em Ciências Veterinárias) - Curso de Pósgraduação em Ciências Veterinárias, Faculdade deVeterinária, UFRGS, 1996.

ALI, J., SHELTON, J.N. Vitrification of preimplantation stages of mouse embryos. Journal of Reproduction and Fertility, Cambridge, England, v.98, p.459-465, 1993a.

ALI, J., SHELTON, J.N. Desing of vitrification solutions for the cryopreservation of embryos. Journal of Reproduction and Fertility, Cambridge, England, v.99, p.471-477, 1993b.

BERTOLINI, M. Sobrevivência in vitro e in vivo de embriões de Mus musculus vitrificados. Porto Alegre, 1994. 229p. Dissertação (Mestrado em Medicina Veterinária) - Curso de Pós-graduação em Ciências Veterinárias, Faculdade de Veterinária, UFRGS, 1994.

CHRISTMANN, L. Congelação de embriões de Mus musculus pelos métodos de vitrificação e rápido: influência da indução à cristalização intracelular (seeding). Porto Alegre, 1989. 100p. Dissertação (Mestrado em Medicina Veterinária) - Curso de Pós-graduação em Medicina Veterinária, Faculdade de Veterinária, UFRGS, 1989.

DINNYÉS, A., WALLACE, G.A., RALL, W.F. Effect of genotype on the efficiency of mouse embryo cryopreservation by vitrification or slow freezing methods. Molecular Reproduction and Development, New York, v.40, p.429435, 1995.

DOBRINSKY, J.R. Cellular approach to cryopreservation of embryos. Theriogenology, Los Altos, California, v.45, p.17$26,1996$.

DULBECCO, R., VOGT, M. Plaque formation and isolation of pure lines with poliomyelits viruses. Journal of Embryology and Experimental Morphology, New York, v.99, p.167-182, 1954.

HÖTZEL, M.J. Influência da temperatura de equilíbrio nas soluções crioprotetoras sobre a sobrevivência in vivo de embriões Mus musculus vitrificados. Porto Alegre, 1991. 83p. Dissertação (Mestrado em Medicina Veterinária) - Curso de Pós-graduação em Medicina Veterinária, Faculdade de Veterinária, UFRGS, 1991.

KASAI, M., KOMI, J.H., TAKAKAMO, A. et al. A simple method for mouse embryo cryopreservation in a low toxicity vitrification solution whithout appreciable loss of viability. Journal of Reproduction and Fertility, Cambridge, England, v.89, p.91-97, 1990

KASAI, M., HAMAGUCHI, S.E., ZHU, T. et al. High survival of rabbit morulae after vitrification in an ethylene glycol-based solution by a simple method. Biology of Reproduction, v.46, p.1042-1046, 1992a.
KASAI, M., NISHIMORI, M., ZHU, T. et al. High survival of mouse morulae after vitrification in an ethylene glycol-based solution after exposure to the solution at various temperatures. Biology of Reproduction, v.47, p.1134-1139, 1992 b.

LOPES, R.F.F. Congelação ultra-rápida e vitrificação de embriões de Mus musculus. Porto Alegre, 1989. 134p. Dissertação (Mestrado em Medicina Veterinária) - Curso de Pós-graduação em Medicina Veterinária, Faculdade de Veterinária, UFRGS, 1989.

MAHMOUDZADEH, A.R., VAN SOOM, A., BOLS, P. et al. Optimization of a simple vitrification procedure for bovine embryos produced in vitro: effect of development stage, twostep addition of cryoprotectant and sucrose dilution on embryonic survival. Journal of Reproduction and Fertility, Cambridge, London, v.103, p.33-39, 1995.

OVERSTRÖM, E.W, DUBY, R.T, DOBRINSKY, J.M. et al. Citoskeletal damage in vitrified or frozen bovine embryos. Theriogenology, Los Altos, California, v.39, p.276, 1993. (Abstract).

PALHA, M.D.C. Congelamento em etapas e vitrificação de mórulas e blastocistos de Mus musculus. Recife, 1992. 375p. Dissertação (Mestrado em Medicina Veterinária) Curso de Mestrado em Medicina Veterinária, Faculdade de Veterinária, UFRPE, 1992

RALL, W.F., WOOD, M.J., KIRBY, C. et al. Development of mouse embryos cryopreserved by vitrification. Journal of Reproduction and Fertility, Cambridge, England, v.80, p.499-504, 1987.

RALL, W.F. Factors affecting the survival of mouse embryos cryopreserved by vitrification. Cryobiology, San Diego, California, v.24, p.387-402, 1987.

ROBERTSON, E.J. Using embrionic stem cells to introduce mutations into the mouse germ line. Biology of Reproduction, v.44, p.238-245, 1991.

SCHMIDT, P.M., LIN, X., BROWN, G.A. et al. The genotypic variability of banking embryos from mouse models in the animal genetic resource. Theriogenology, Los Altos, California, v.45, p.171, 1996. (Abstract).

SZÉLL, A., SHELTON, J.N. Role of equilibration before rapid freezing of mouse embryos. Journal of Reproduction and Fertility, Cambridge, London, v.78, p.699-703, 1986.

TACHIKAWA, S., OTOI, T., KONDO, S. et al. Successful vitrification of bovine blastocysts, derived by in vitro maturation and fertilization. Molecular Reproduction and Development, Cambridge, v.34, p.266-271, 1993.

WHITTINGHAM, D.G. Viability assays for mammalian ova. Cryobiology, San Diego, v.15, p.245-248; 1978.

ZHU, S.E., KASAI, M., OTOGE, H. et al. Cryopreservation of expanded mouse blastocysts by vitrification in ethylene glycol-based solutions. Journal of Reproduction and Fertility., v.98, p.139-145, 1993. 